Discrete Comput Geom 35:481-491 (2006)

DOI: $10.1007 / \mathrm{s} 00454-005-1223-\mathrm{x}$

Geometry

\title{
Bar-Magnet Polyhedra and NS-Orientations of Maps*
}

\author{
Bojan Mohar \\ Department of Mathematics, University of Ljubljana, \\ 1000 Ljubljana, Slovenia \\ bojan.mohar@uni-lj.si
}

\begin{abstract}
At the CCCG 2001 open-problem session [2], J. O'Rourke asked which polyhedra can be represented by bars and magnets. This problem can be phrased as follows: which 3-connected planar graphs may have their edges directed so that the directions "alternate" around each vertex (with one exception of nonalternation if the degree is odd). In this note we solve O'Rourke's problem and generalize it to arbitrary maps on general surfaces. Obstructions to the existence of such orientations can be expressed algebraically by a new homology invariant of perfect matchings in the related graph of cofacial odd vertices.
\end{abstract}

\section{Bar-Magnet Polyhedra}

A toy called "Roger's Connection" (produced by the company with the same name as the toy) provides a collection of magnetic bars and steel balls that can be used to construct polyhedra. The structures are most stable when around each vertex (a steel ball), the North and South poles of magnetic bars meeting at that vertex are alternating. This toy motivated the definition of bar-magnet polyhedra as those 3-connected plane graphs whose edges can be directed so that the directions "alternate" around each vertex, where one nonalternation is allowed if the degree of the vertex is odd.

Let $G$ be a map, i.e., a graph that is 2-cell embedded in some surface. By a 2-cell embedding we mean an embedding of a graph into a closed surface such that every face is homeomorphic to an open disk (but it may happen that the closure of a face in the surface is not a disk due to multiple occurrences of vertices or edges on the boundary of that face). We refer to [4] for basic definitions concerning maps and graphs on surfaces. An NS-orientation of $G$ is an orientation of the edges so that in the clockwise order

\footnotetext{
* This research was supported in part by the Ministry of Education, Science and Sport of Slovenia, Research Program P1-0507-0101. The author is currently at the Department of Mathematics, Simon Fraser University, Burnaby, British Columbia, Canada V5A 1S6.
} 
around every vertex $v$, the incoming and outgoing edges alternate, except when $\operatorname{deg}(v)$ is odd when one violation of this condition is allowed. We say that the pair of consecutive edges in the clockwise order around $v$, at which nonalternation occurs, is the exceptional transition at $v$. We say that $G$ is NS-orientable if it admits an NS-orientation.

At the open-problem session at the 13th Canadian Conference on Computational Geometry in 2001 [2], O'Rourke asked which 3-connected planar maps are NS-orientable. At the corresponding web page of open problems, maintained by Demaine et al. [1], it is noted that after the presentation of the problem, Biedl proved that the polyhedron formed by gluing together two tetrahedra with congruent bases is not NS-orientable. Demaine proved that a polyhedron, all of whose vertices have even degree, is NS-orientable: the graph has a face 2-coloring, and the edges of the faces of color 1 can be oriented counterclockwise, which then orients each face of color 2 clockwise. Demaine also observed that every cubic graph with a perfect matching admits an NS-orientation: orient every cycle in the complement of the perfect matching cyclically and orient the edges of the perfect matching arbitrarily.

In this note we obtain a complete solution to O'Rourke's problem by providing a simple characterization of NS-orientability of planar maps (see Corollary 2.3). We also obtain a generalization of this result to arbitrary maps on general surfaces. Obstructions to the existence of NS-orientations depend on certain perfect matchings in an associated graph of cofacial odd vertices and give rise to a new homology invariant of perfect matchings.

\section{NS-Orientations and Matchings}

By a map we mean a connected graph together with a 2-cell embedding in some closed surface. Underlying graphs of maps are allowed to have loops and multiple edges. If $\Sigma$ is a surface, the number $g=2-\chi(\Sigma)$ is called the Euler genus of $\Sigma$, where $\chi(\Sigma)$ denotes the Euler characteristic of the surface.

Maps in orientable surfaces can be described (up to a homeomorphism) in a purely combinatorial way by specifying the local rotation around each vertex. First, we fix a global orientation on the surface, and then we consider, for each vertex $v$, the clockwise cyclic permutation of edges emanating from $v$, as seen on the surface. This permutation is called the local rotation around $v$. Having such a rotation for each vertex, we speak of a rotation system which determines the original embedding up to a homeomorphism. It is easy to see that local rotations describe anticlockwise traversals of boundaries of faces: follow an edge and when arriving at its end, take the edge following it in the local rotation at that vertex, follow that edge, etc. In this way we obtain a collection of closed walks in the graph, called the facial walks, since they correspond to traversals of boundaries of the faces. The surface can be reconstructed from these by taking the graph, and pasting a disk on each facial walk.

If the surface of the map is nonorientable, then we need another ingredient in order to describe the map. For each edge $e$ we provide the value $\lambda(e) \in\{-1,1\}$, called the signature of $e$. We may assume that the signatures on an arbitrary spanning tree $T$ are all equal to 1 . Then the edge $e \in E(G) \backslash E(T)$ has a negative signature if and only if the cycle in $T+e$ is orientation-reversing on the surface. It means that, when traversing the edge $e$, we have to change from "clockwise" to "anticlockwise." By accounting for this, 
facial walks (face boundaries) are determined in a similar manner as in the orientable case. Again, we refer the reader to [4] for more details.

If $G$ is a map, we define the dual map $G^{*}$ as follows. In each face $F$ of $G$ we put a vertex of $G^{*}$, and for every edge $e$ of $G$, we draw an edge of $G^{*}$ connecting the two faces incident with $e$. (It may happen that $e$ appears twice on the boundary of the same facial walk, in which case the dual edge is a loop.) In this way the local rotation around the vertex of $G^{*}$ in $F$ coincides with the cyclic sequence of the edges on the facial walk of $F$ in $G$.

A graph (or a map) is Eulerian if all its vertices have even degree.

Let $G$ be an Eulerian map on a surface $S$ and let $G^{*}$ be its dual map. Suppose that the embedding of $G^{*}$ is represented by local rotations and signatures. We say that $G$ is orientably partitionable if the faces of $G$ can be partitioned into two classes $F^{\prime} \cup F^{\prime \prime}$ such that two adjacent faces are in the same class if and only if the corresponding edge in $G^{*}$ has a negative signature. It is easy to see that $G$ is orientably partitionable if and only if for every cycle $C$ in the dual $G^{*}$, the number of edges on $C$ with positive signature is even, i.e., the number of edges with a negative signature has the same parity as the length of $C$. Alternatively, every even cycle of $G^{*}$ is orientation preserving and every odd cycle is orientation reversing on the surface. This implies that being orientably partitionable is independent of the choice of the rotation-signature representation of $G^{*}$. In particular, if the surface $S$ is orientable, then all signatures can be taken to be positive, and being orientably partitionable is equivalent to being face 2 -colorable.

Proposition 2.1. Let $G$ be an Eulerian map. Then $G$ is NS-orientable if and only if it is orientably partitionable.

Proof. Under any NS-orientation, facial walks are directed closed walks. Adjacent facial walks are oppositely oriented when compared with respect to a common edge. In an orientable surface, this means that faces are either positively or negatively oriented, and this yields a bipartition of the faces. If the surface is nonorientable, then cycles of $G^{*}$ of even length behave similarly as on orientable surfaces and they must be orientation preserving, while cycles of odd length in $G^{*}$ must be orientation reversing. These facts easily yield the stated equivalence.

Let $G$ be a map. We say that distinct vertices of $G$ are cofacial if they lie on a common facial walk. The cofaciality graph $R=R(G)$ of $G$ is the graph whose nodes are the vertices of odd degree in $G$, two of them being adjacent if they are cofacial in $G$.

Theorem 2.2. A map $G$ has an NS-orientation if and only if its cofaciality graph $R(G)$ has a perfect matching $M$ such that $G$ can be extended to an orientably partitionable map in the same surface, whose underlying graph is $G+M$.

Proof. Suppose that we have an NS-orientation of $G$. Let $F$ be a facial walk. When we traverse the facial walk $F$, the edges all head in the same direction until an exceptional transition is met. From that point on, all edges head in the other direction until the next exceptional transition is reached, when the direction changes again. This implies 
Claim 1. Every facial walk has an even number of exceptional transitions.

Let $G^{\prime}$ be the map obtained from $G$ by adding a new vertex $v_{F}$ in every face $F$ with at least one exceptional transition and joining $v_{F}$ to all vertices in $F$ corresponding to exceptional transitions.

Claim 2. $G^{\prime}$ is an Eulerian graph and the NS-orientation of $G$ uniquely extends to an $N S$-orientation of $G^{\prime}$. Under this orientation, all faces of $G^{\prime}$ are directed closed walks, and hence $G^{\prime}$ is orientably partitionable.

We consider a facial walk $F=v_{1} e_{1} v_{2} e_{2} \cdots v_{k} e_{k} v_{1}$ where $v_{i}$ and $e_{i}$ are consecutive vertices and edges (respectively) on $F$. If the edges $e_{i-1}$ and $e_{i}$ form an exceptional transition at the vertex $v_{i}$, then $G^{\prime}$ has the edge $e_{i}^{\prime}=v_{F} v_{i}$ that is inserted between $e_{i-1}$ and $e_{i}$ in the local clockwise rotation around $v_{i}$ on the surface. Clearly, there is a unique way of orienting $e_{i}^{\prime}$ so that none of the transitions $e_{i-1}, e_{i}^{\prime}$ and $e_{i}^{\prime}, e_{i}$ is exceptional. It is also clear that if the exceptional transition of $G$ in $F$ that immediately follows the transition at $v_{i}$ occurs at $v_{j}$, then the orientation (at $v_{F}$ ) of the inserted edge $e_{j}^{\prime}=v_{F} v_{j}$ is opposite to that of $e_{i}^{\prime}$. This shows that all exceptional transitions of $G$ disappear in $G^{\prime}$ and that no exceptional transitions arise at new vertices $v_{F}$. In particular, all vertices of $G^{\prime}$ have even degree, i.e., $G^{\prime}$ is Eulerian. By Proposition 2.1, $G^{\prime}$ is orientably partitionable. This completes the proof of Claim 2.

Let us now consider a vertex $v_{F}$ of $G^{\prime}$. Suppose that its neighbors on $F$ in the clockwise order are $u_{1}, \ldots, u_{2 k}$. Let $M_{F}$ be the set of edges $u_{1} u_{2}, \ldots, u_{2 k-1} u_{2 k}$. Finally, let $M$ be the union of all $M_{F}$, where $F$ is any face of $G$. Since each odd vertex of $G$ has precisely one exceptional transition (and vertices of even degree have none), $M$ is a perfect matching in $R$. Since $G^{\prime}$ is orientably partitionable, it is clear that the map $\tilde{G}$ obtained from $G$ by adding all edges of every $M_{F}$ in $F$ (following the boundary of $F$ ) is also orientable partitionable. This completes the first part of the proof.

The proof of the converse implication is essentially the reverse of the above proof, so we omit it.

Corollary 2.3. Let $G$ be a graph that is 2-cell embedded in the plane or the 2-sphere, and let $R$ be its cofaciality graph. Then $G$ has an NS-orientation if and only if $R$ has a perfect matching.

Proof. Suppose that $R$ has a perfect matching $M$. If $e=u v \in M$, there is a face in which $u$ and $v$ both appear. For every $e \in M$ choose one such face. For a given face $F$ of $G$, let $M_{F}$ be all edges in $M$ that have selected $F$ as their face of cofaciality. Let $u_{1}, \ldots, u_{2 k}$ be the endvertices of the edges in $M_{F}$ in the order as they appear on the facial walk of $F$. Let $M_{F}^{\prime}=\left\{u_{1} u_{2}, \ldots, u_{2 k-1} u_{2 k}\right\}$ and let $M^{\prime}$ be the union of all $M_{F}^{\prime}$. Clearly, $M^{\prime}$ is a matching of $R$ and the embedding of $G$ can be extended to an embedding of $\tilde{G}=G+M^{\prime}$, yielding an Eulerian map. A well-known consequence of simple connectivity is that every Eulerian map in a simply connected surface is face 2-colorable. So it is $\tilde{G}$, and we are done by Theorem 2.2 . 


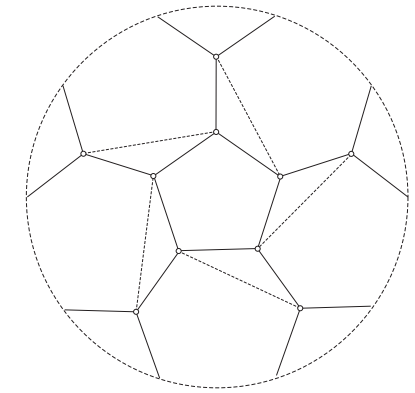

Fig. 1. An orientably partitionable extension of the Petersen graph in the projective plane.

Since the existence of a perfect matching is polynomially checkable, Corollary 2.3 provides a good characterization in the sense of Edmonds. This is the best one can hope for.

For instance, if we have a planar map with precisely two vertices of odd degree, then an NS-orientation exists if and only if the two odd vertices are cofacial.

\section{Maps on General Surfaces}

An example, the Petersen graph on the projective plane, is shown in Fig. 1. The dotted edges form a perfect matching of $R$ and it is easy to check that the extended map is orientably partitionable.

As mentioned above, the existence of a perfect matching is polynomially solvable, but perfect matchings satisfying additional conditions may be harder to detect. Nevertheless, since some kind of parity is involved, we believe that Theorem 2.2 yields a good characterization also for more general maps. This belief is partially supported by our subsequent results (see Corollary 3.3), at least if we consider maps on a fixed surface.

It is worthwhile noting that the same pair of vertices can appear on more than one facial walk, and can appear more than once on the same facial walk. In such a case we get multiple edges in $R$. Different edges in $\tilde{G}$ joining the same pair of vertices but corresponding to distinct possibilities of cofaciality may give rise to different partiteness behavior of $\tilde{G}$. See Fig. 2 for an example of a map on the torus, where the choice of the

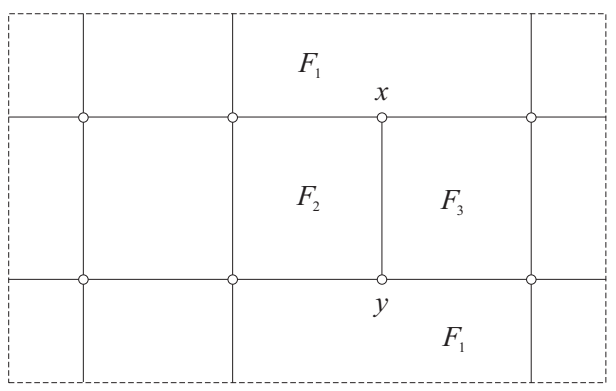

Fig. 2. A toroidal map with two extensions. 
edge $x y$ drawn inside the face $F_{1}$ gives rise to an orientably partitionable map, while its inclusion in one of the faces $F_{2}$ or $F_{3}$ does not give that. Of course, in the case of planar maps, such a distinction is not necessary since all possibilities are equally good as shown by Corollary 2.3.

The above example shows that it may be helpful to introduce the extended cofaciality graph $\tilde{R}=\tilde{R}(G)$ that will capture not only the combinatorial but also geometric information about cofaciality of odd vertices. The vertices of $\tilde{R}$ are the vertices of odd degree in $G$. Suppose that $u, v$ are distinct vertices of $\tilde{R}$. If $u$ appears in a facial walk $F$ and $v$ appears in the same facial walk, then we have an edge joining $u$ and $v$ in $\tilde{R}$ for every such pair of common appearances. Observe that a vertex can appear more than once in a facial walk. We say that this edge corresponds to the face $F$ and to appropriate appearances of $u, v$ in $F$. Let $v_{F}$ be a point on the surface in the interior of the face $F$, and let $S_{1}, \ldots, S_{r}$ be internally disjoint simple arcs joining occurrences of vertices in $F$ with $v_{F}$. If $S_{i}$ and $S_{j}$ correspond to the appearances of $u$ and $v$ which determine an edge $e$ of $\tilde{R}$, then we say that $S_{i} \cup S_{j}$ is a drawing of $e$ in $F$. If $M \subseteq E(\tilde{R})$ is a matching, the drawings of edges in $M$ determine an Eulerian map whose graph consists of $G$ together with edges (and vertices $v_{F}$ ) corresponding to those segments $S_{i}$ that correspond to edges in $M$. We denote this map by $G \dot{+} M$.

We have to introduce another concept, that of $(G F(2)-)$ homology; see any textbook on algebraic topology for a comprehensive treatment, e.g., [3]. A set of edges $E^{\prime}$ of a map $G$ is a 1-cycle if each vertex has even degree in $E^{\prime}$. The set of 1-cycles with the symmetric difference operation + is an Abelian group, denoted by $\mathcal{C}_{1}(G)$. This group can also be viewed as a vector space over $G F(2)$ and is called the cycle space of the graph $G$. If $F$ is a closed walk in $G$, the edges that appear an odd number of times in $F$ form a 1-cycle. For convenience, we denote the 1-cycle corresponding to $F$ by the same symbol $F$.

Two 1-chains $E_{1}, E_{2}$ are homologically equivalent if there is a family of facial walks $F_{1}, \ldots, F_{r}$ of $G$ such that $E_{1}+F_{1}+\cdots+F_{r}=E_{2}$. Being homologically equivalent is an equivalence relation compatible with the symmetric difference of sets. The 1-cycles that are homologically equivalent to the empty set, form a subgroup $\mathcal{B}_{1}(G)$ of $\mathcal{C}_{1}(G)$. The quotient group $H_{1}(G ; G F(2))=\mathcal{C}_{1}(G) / \mathcal{B}_{1}(G)$ is called the homology group of the $\operatorname{map} G$.

Let $B$ be a map in a surface $\Sigma$ such that all faces are of even length. Then we say that $B$ is locally bipartite. Let $H_{1}=H_{1}(B ; G F(2))$ be the first homology group of the map $B$ with coefficients in the group $G F(2)$. For $C \subseteq E(B)$, let $\sigma(C)$ be equal to the parity of the number of edges in $C$ whose signature is positive. Since $\sigma(C)=0$ for the edge set $C$ of any facial walk, $\sigma$ induces a homomorphism

$$
\sigma_{0}: H_{1} \rightarrow G F(2) \text {. }
$$

To relate this homomorphism to our previous thoughts, we observe that an Eulerian map $G$ is orientably partitionable if and only if the homomorphism $\sigma_{0}$ corresponding to the dual map $G^{*}$ is trivial, i.e., $\sigma_{0}\left(H_{1}\right)=\{0\}$.

If $G$ is a map and $M$ is a perfect matching in the extended cofaciality graph $\tilde{R}(G)$, then the map $G_{M}=G \dot{+} M$ is Eulerian. Let $B=G_{M}^{*}$ be its dual map, and let $\sigma_{M}: H_{1}(B ; G F(2)) \rightarrow G F(2)$ be the corresponding homomorphism. We call $\sigma_{M}$ the characteristic mapping of $M$. These definitions yield a reformulation of Theorem 2.2: 
Theorem 3.1. A map $G$ is NS-orientable if and only if its extended cofaciality graph $\tilde{R}(G)$ contains a perfect matching whose characteristic map is trivial.

If $M_{1}, M_{2}$ are perfect matchings of $\tilde{R}$, we may consider their symmetric difference $L=M_{1}+M_{2}$. In $\tilde{R}, L$ is a collection of disjoint cycles. Drawings of these cycles (as defined above) give rise to a collection of closed curves in $\Sigma$. We say that $M_{1}$ and $M_{2}$ are homologous if the collection of these curves is 0-homologous on the surface (with respect to $G F(2)$-homology). If $L=M_{1}+M_{2}$ and $L^{\prime}=M_{2}+M_{3}$ are both 0-homologous on the surface, so is $L+L^{\prime}=M_{1}+M_{3}$. This implies that the homology of perfect matchings is an equivalence relation.

Lemma 3.2. Perfect matchings $M_{1}, M_{2}$ of $\tilde{R}(G)$ are homologous if and only if their characteristic mappings are the same.

Proof. If $M_{1}$ and $M_{2}$ are homologous, then $L=M_{1}+M_{2}$ is 0-homologous on the surface. Therefore, every simple closed curve crosses edges of $L$ an even number of times. In particular, this holds for cycles in dual maps $G_{M_{1}}^{*}, G_{M_{2}}^{*}$, and, consequently, $\sigma_{M_{1}}=\sigma_{M_{2}}$.

On the other hand, if $L$ is not 0 -homologous, then there is a cycle in the dual map that intersects $L$ an odd number of times. If $\gamma$ is the homology class of that cycle, then $\sigma_{M_{1}}(\gamma) \neq \sigma_{M_{2}}(\gamma)$. This completes the proof.

At this point it is clear why the cofaciality graph $\tilde{R}(G)$ did not need geometric information when considering maps in simply connected surfaces. This is because all perfect matchings are homologous and since every homomorphism $\{0\} \rightarrow G F(2)$ is trivial.

If $\Sigma$ is a surface of Euler genus $g$, then $H_{1}$ is isomorphic to $G F(2)^{g}$. This gives

Corollary 3.3. A map on a surface of Euler genus $g$ has at most $2^{g}$ distinct homology classes of perfect matchings for cofacial odd vertices.

Sometimes, checking if extensions are orientably partitionable can be overridden. For instance, if the graph $G$ of the map is cubic (all vertices have degree 3), then NSorientability is independent of the embedding of $G$ since NS-orientations correspond to orientations of edges such that no vertex is a sink (all edges incoming) or a source (all edges outgoing). Therefore, it makes sense to speak of NS-orientability of the graph. The same holds for subcubic graphs in which all vertices have degree at most 3. Recall that we allow loops and multiple edges.

Proposition 3.4. Every subcubic graph is NS-orientable.

Proof. The proof is by induction on the number of edges. If $G$ has a vertex $v$ of degree 1 , let $u$ be its neighbor and let $G_{1}=G-v$. By the induction hypothesis, $G_{1}$ has an NS-orientation. If $u$ has at least one incoming edge under such an orientation, then we orient the edge $u v$ in the direction from $u$ to $v$; otherwise from $v$ to $u$. Clearly, this gives 
rise to an NS-orientation of $G$. If $G$ has a vertex $v$ of degree 2, and $u v, v w$ are its incident edges, then we apply induction to the graph $G_{1}=(G-v)+u w$. An NS-orientation of $G_{1}$ clearly gives rise to one in $G$.

Otherwise, $G$ is a cubic graph. If $G$ has a cutedge $u v$, we apply induction to $G-u v$ and orient the edge $u v$ arbitrarily. Finally, suppose that $G$ has no cutedges. Then $G$ has a perfect matching $M$ by a well-known theorem of Petersen. The graph $G-M$ consists of a collection of disjoint cycles. By orienting the edges of each such cycle consistently with a chosen direction on the cycle, and orienting edges in $M$ arbitrarily, an NS-orientation is obtained. This completes the proof.

When considering embedded cubic graphs, we can say much more.

Theorem 3.5. Let $G$ be a map in a surface $\Sigma$ of Euler genus $g$. If every vertex of $G$ has degree at most 3 and $G$ is not a cycle that is 2-cell embedded in the projective plane, then for every homomorphism $\sigma: H_{1}(\Sigma ; G F(2)) \rightarrow G F(2)$ there exists a perfect matching in the extended cofaciality graph $\tilde{R}(G)$, whose characteristic map is equal to $\sigma$. In other words, perfect matchings of all possible $2^{g}$ homology classes exist.

The proof of Theorem 3.5 is deferred until the end of this section.

Observe that the map whose graph is the cycle in the projective plane has only one matching of $\tilde{R}$ (the empty matching) and its characteristic map is the trivial homomorphism.

A graph is called a $\Theta$-graph if it consists of three internally disjoint paths joining two vertices.

Lemma 3.6. If the graph of $G$ is a $\Theta$-graph, then $G$ satisfies the conclusion of Theorem 3.5.

Proof. $\Theta$-graphs admit precisely four nonequivalent embeddings, one in the plane, the projective plane, the Klein bottle, and the torus; see Fig. 3 for the last three of these cases.

We consider the $\Theta$-graph map on the torus. As the generators for the fundamental group (and consequently for $H_{1}$ ), we select the horizontal and the vertical simple closed curves $\alpha$ and $\beta$ (respectively) with respect to the presentation in Fig. 3(c). By taking the four possible matching edges of $\tilde{R}(G)$ which are shown in Fig. 4, the resulting values

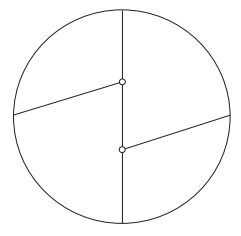

(a)

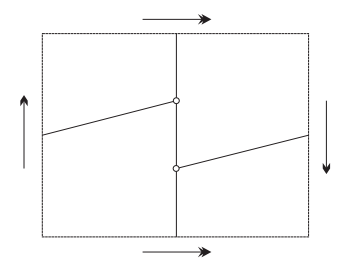

(b)

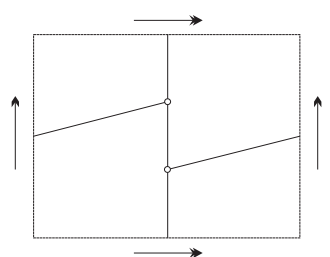

(c)

Fig. 3. Three maps with a $\Theta$-graph. 


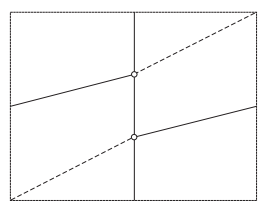

00

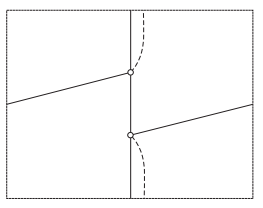

01

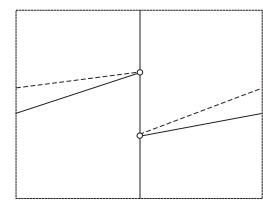

10

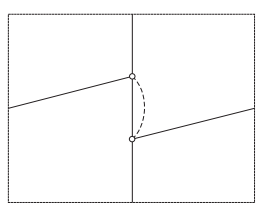

11

Fig. 4. Four nonhomologous extensions.

of their characteristic maps on $\alpha$ and $\beta$ are $00,01,10$, and 11 , respectively. This proves the claim in the case of the toroidal map. The proof for the Klein bottle is essentially the same, while the planar and the projective planar cases are obvious. The details are left to the reader.

Lemma 3.7. The dumbbell map on the Klein bottle shown in Fig. 5(a) satisfies the conclusion of Theorem 3.5 .

Proof. Four extensions with distinct characteristic maps are shown by dotted edges in Fig. 5(b).

We are ready for the proof of Theorem 3.5.

Proof of Theorem 3.5. The proof is by induction on the number of edges of $G$. If $G$ has a vertex of degree 2, the reduction is exactly the same as in the proof of Proposition 3.4. The same proof can be followed if $G$ has a vertex $v$ of degree 1 and $G-v$ is not the cycle in the projective plane. However, in the latter case it is easy to check that the theorem holds.

Suppose now that all vertices of $G$ have degree 3. If $G$ is not 2-connected, it contains a cutedge $e=u v$. The embedding of $G$ gives rise to two maps $G_{1}, G_{2}$ whose graphs are the two components of $G-e$. Vertices $u \in V\left(G_{1}\right)$ and $v \in V\left(G_{2}\right)$ become vertices of degree 2. If none of $G_{1}, G_{2}$ is just the cycle in the projective plane, then we apply the induction hypothesis for $G_{1}$ and $G_{2}$. For every perfect matching $M_{1}$ in $\tilde{R}\left(G_{1}\right)$ and $M_{2}$ of $\tilde{R}\left(G_{2}\right)$, let $M$ be the perfect matching of $\tilde{R}(G)$ consisting of $M_{1}, M_{2}$ and the edge $u v$ (embedded so that it is homotopic to the edge $e$ in $G$ ). By the induction hypothesis,

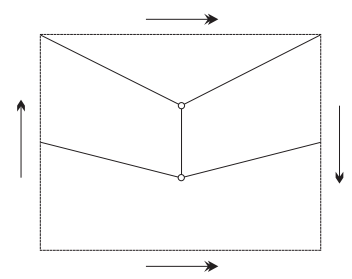

(a)

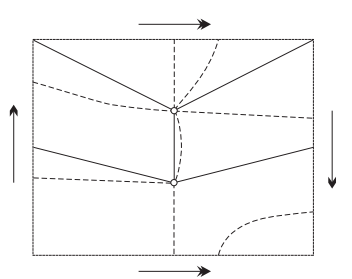

(b)

Fig. 5. The dumbbell map on the Klein bottle. 
perfect matchings of $\tilde{R}\left(G_{i}\right)$ give rise to all possible $2^{g_{i}}$ characteristic mappings (where $g_{i}$ is the Euler genus of $\left.G_{i}\right), i=1,2$. Since the Euler genus of $G$ is $g=g_{1}+g_{2}$, it is now easy to see that all combinations of $M_{1}$ and $M_{2}$ give rise to $2^{g}$ distinct characteristic maps corresponding to perfect matchings of $\tilde{R}(G)$.

If $G_{1}$ and $G_{2}$ are both cycles in the projective plane, then $G$ is the dumbbell map in the Klein bottle, and we apply Lemma 3.7. If $G_{1}$ is the cycle in the projective plane but $G_{2}$ is not, we proceed in a similar way as above, except that we combine every perfect matching $M_{2}$ of $\tilde{R}\left(G_{2}\right)$ first with the edge $u v$ embedded homotopic to $e$ in $G$, and secondly with the edge $u v$ embedded so that it first follows $e$ from $v$ towards $u$ and then goes across the crosscap, following the cycle $G_{1}$. The resulting matchings give rise to $2 \cdot 2^{g_{2}}=2^{g}$ distinct characteristic maps.

From now on we may assume that $G$ is 2-connected. Let $e=u v$ be an edge of $G$ and consider the induced map $G^{\prime}=G-e$. If $G$ is a $\Theta$-graph, we are done by Lemma 3.6. So, we may assume that $G^{\prime}$ is not a cycle, and we can apply the induction hypothesis to $G^{\prime}$. Observe that vertices $u, v$ are of degree 2 in $G^{\prime}$.

We distinguish three subcases outlined below. Since the choice of $e$ is arbitrary, we may assume (in (2) and (3)) that no edge of $G$ can be chosen so that one of the previous cases would be obtained.

(1) The edge uv belongs to two distinct facial walks in $G$. In this case the Euler genus of $G^{\prime}$ is the same as that of $G$. Let $F$ be the facial walk of $G^{\prime}$ that is the combination of the two facial walks of $G$ containing $u v$. To every perfect matching $M^{\prime}$ of $\tilde{R}\left(G^{\prime}\right)$, we associate a perfect matching $M$ of $\tilde{R}(G)$ as follows. If $e^{\prime} \in M^{\prime}$ corresponds to a face different from $F$, then we include $e^{\prime}$ in $M$. Let $e_{1}^{\prime}, \ldots, e_{t}^{\prime}$ be the edges in $M^{\prime}$ that correspond to $F$. The problem is that these edges may not be edges of $\tilde{R}(G)$. Consider the occurrences of the endpoints of $e_{1}^{\prime}, \ldots, e_{t}^{\prime}$ on $F$; denote them by $v_{1}, \ldots, v_{2 t}$ in the order in which they appear in $F$ and such that $v$ appears between $v_{2 t}$ and $v_{1}$ and that $u$ appears between $v_{j}$ and $v_{j+1}$, where the considered appearances of $u$ and $v$ correspond to the removed edge $u v$. If $j$ is even, then we add to $M$ the following edges of $\tilde{R}(G): v v_{1}, \ldots, v_{j} u$ and $v_{j+1} v_{j+2}, \ldots, v_{2 t-1} v_{2 t}$. If $j$ is odd, then we add to $M$ the edges $v v_{1}, \ldots, v_{j-1} v_{j}$ and $u v_{j+1}, \ldots, v_{2 t-1} v_{2 t}$.

(2) The edge uv belongs to a single facial walk $F$ in $G$ and is traversed on $F$ twice in the same direction. In this case the Euler genus of $G^{\prime}$ decreases by 1 . Excluding the possibility of (1), $F$ is the only facial walk of $G$ and hence $G^{\prime}$ also has only one facial walk, which we denote by $F^{\prime}$. To every perfect matching $M^{\prime}$ of $\tilde{R}\left(G^{\prime}\right)$, we associate two perfect matchings $M_{1}, M_{2}$ of $\tilde{R}(G)$. For $M_{1}$, we just combine $M^{\prime}$ with the edge $u v$ embedded in $G$ homotopic to the removed edge. In $M_{2}$ we add $u v$ embedded across the face $F$ (joining the appearance of $u$ at the first traversal of $e$ and the appearance of $v$ at the second traversal of $e$ in $F$ ).

(3) The edge uv belongs to a single facial walk $F$ in $G$ and is traversed twice in the different direction. In this case the Euler genus of $G^{\prime}$ decreases by 2 and $F$ gives rise to two new facial walks $F_{1}, F_{2}$. To every perfect matching $M^{\prime}$ of $\tilde{R}\left(G^{\prime}\right)$, we now associate four perfect matchings $M_{1}, M_{2}, M_{3}, M_{4}$ of $\tilde{R}(G)$. For each of them we add to $M^{\prime}$ the edge $u v$ embedded in $G$ within the face $F$ in a different manner, similarly as shown in the example in Fig. 4. More precisely, let $v_{\alpha}$ and 
$u_{\alpha}$ be the appearances of $v$ and $u$ in $F$ at the first traversal of $e$, and let $v_{\beta}$ and $u_{\beta}$ be the their appearances at the second traversal of $e$. Since $F$ is the only face, there exists a third appearance $v_{\gamma}$ of $v$ and another appearance $u_{\gamma}$ of $u$. Then we take $M_{1}=M^{\prime} \cup\left\{u_{\alpha}, v_{\alpha}\right\}, M_{2}=M^{\prime} \cup\left\{u_{\alpha}, v_{\beta}\right\}, M_{3}=M^{\prime} \cup\left\{u_{\alpha}, v_{\gamma}\right\}$, and $M_{4}=M^{\prime} \cup\left\{u_{\gamma}, v_{\alpha}\right\}$.

By applying the induction hypothesis to $G^{\prime}$, it is easy to see that in every one of the three above possibilities, the obtained perfect matchings of $\tilde{R}(G)$ give rise to $2^{g}$ distinct characteristic maps.

\section{References}

1. Erik D. Demaine, Joseph S. B. Mitchell, and Joseph O'Rourke, The open problems project, Problem 32. http://cs.smith.edu/ orourke/TOPP/

2. Erik D. Demaine and Joseph O'Rourke, Open problems from CCCG 2001, in Proceedings of the 14th Canadian Conference on Computational Geometry, August 2002. http: / /www. cs. uleth.ca/ $\sim_{\text {wismath/cccg/papers/open.pdf. }}$

3. P. J. Giblin, Graphs, Surfaces and Homology. An Introduction to Algebraic Topology, second edition, Chapman \& Hall, London, 1981.

4. Bojan Mohar and Carsten Thomassen, Graphs on Surfaces, Johns Hopkins University Press, Baltimore, MD, 2001.

Received September 17, 2004, and in revised form September 2, 2005. Online publication January 20, 2006. 